\title{
Lectura y Escritura. Autopercepción del Desempeño en Estudiantes Universitarios
}

\author{
Reading and Writing in University Students. Self-perceived Performance
}

\author{
Luis Ángel Roldán ${ }^{1}$ y Verónica Zabaleta ${ }^{2}$
}

\begin{abstract}
Resumen
En este trabajo se examina la autopercepción del desempeño en lectura y escritura de estudiantes de Psicología de la UNLP (Argentina). Se inscribe en las investigaciones sobre la "alfabetización académica" y surge de la preocupación docente sobre la insuficiencia de habilidades lingüísticas académicas de los alumnos y consecuentemente del alto porcentaje de deserción universitaria. En un estudio descriptivo transeccional se examinaron 50 alumnos de $1^{\circ}$ año y 40 de $5^{\circ}$ a través de un cuestionario diseñado para detectar la presencia/ausencia de la autopercepción de dificultades, el tipo de dificultades detectadas y la atribución causal. Se identificaron semejanzas/diferencias en la autopercepción de los participantes en esos dos grupos: los ingresantes perciben mayores dificultades. Se discuten los alcances de esos resultados en relación con las problemáticas abordadas y su importancia en el diseño de estrategias de intervención que favorezcan el desempeño exitoso en los estudios universitarios.
\end{abstract}

Palabras clave: autopercepción, lectura, escritura, alumnos universitarios

\begin{abstract}
This study examines the self-perception of Psychology Students (UNLP-Argentina) performance as readers and writers. It is carried out within the framework of research on "academic literacy" and stems from instructors' concerns about the inadequacy of academic language skills of students and consequently the high percentage of college dropout. A transactional descriptive study examined 50 first year students and 40 fifth year students and was conducted via a questionnaire designed to detect the presence/absence of selfperception of difficulty, the types of perceived difficulties, and causes for such difficulties. Similarities/differences in participants' self-perception in these two groups were identified: 1 st year students perceive greater difficulties. The conclusions discuss the implications of these results in terms of the issues addressed and their importance in the design of intervention strategies to promote successful performance in college.
\end{abstract}

Keywords: self-perception, reading, writing, university students

\footnotetext{
El trabajo que se presenta corresponde a una investigación radicada en el Instituto de Investigaciones en Psicología (InIPsi), de esa misma Facultad. Dirección postal: Calle 51 entre 123 y 124, 3er. Piso, Instituto de Investigaciones. Facultad de Psicología. Ensenada. Provincia de Buenos Aires. Argentina. (C.P. 1925). Teléfonos: +54 (0221) 482-4415 | 482-5931 | 482-8457 | 483-3933 | 483-4601 | 483-4871. Interno 135

${ }^{1}$ Profesor en Psicología, docente de la Cátedra de Psicología Educacional. Facultad de Psicología. Universidad Nacional de La Plata, La Plata, Argentina. angelroldan1990@gmail.com

${ }^{2}$ Doctora en Psicología y Especialista en procesos de aprendizaje del lenguaje escrito y sus trastornos. Universidad Nacional de La Plata, La Plata, Argentina. veronicazabaleta@gmail.com
} 


\section{Introducción}

Este trabajo, destinado a examinar la autopercepción de estudiantes de psicología como lectores y escritores, se inscribe en el marco de los estudios que enfatizan la importancia de la denominada "alfabetización académica". Ella implica la interacción textual con textos progresivamente complejos, propios de la comunidad disciplinar de la que se trate (Piacente, 2012a). Surge, por un lado, de la preocupación creciente de los docentes sobre la insuficiencia de las "habilidades lingüísticas académicas" de los estudiantes (August \& Shanahan, 2008; Halliday \& Martin, 1993; Schleppegrell \& Colombi, 2002) $\mathrm{y}$ de los resultados de otras investigaciones realizadas en el país (Piacente 2012b; Piacente \& Tittarelli, 2008). Por otro, particularmente en Argentina, del problema de la deserción universitaria, que alcanza porcentajes altos y comprende, entre otras razones múltiples y complejas, el manejo adecuado de la lectura y la escritura, de modo tal que su dominio resulta un factor crucial para la permanencia de los estudiantes (Olave-Arias, Rojas García, \& Cisneros-Estupiñán, 2013). De hecho, el control sobre el lenguaje académico es un requisito para el éxito en tareas desafiantes y complejas, como la lectura de libros de autor o la escritura de monografías, informes, artículos de investigación y de revisión. Se trata en ambos casos de actividades que forman parte de los aprendizajes del tercer nivel y posteriormente de las que corresponden al quehacer académico/profesional (Carlino, 2005, Snow \& Ucelli, 2009).

\section{Comprensión y producción textual. Papel de la metacognición en la lectura y la escritura}

La comprensión de un texto se refiere a una a capacidad cognitiva compleja que implica construir una representación mental coherente del contenido del mismo. Para ello es necesario sostener, procesar y almacenar la información que proviene del texto e integrarla con los conocimientos previos que ya posee el lector (conocimientos lingüísticos, conocimientos generales del mundo y conocimientos específicos de dominio) (Abusamra, Casajús, Ferreres, Raiter; De Beni, \& Cornoldi, 2011; Abusamra, Ferreres, Raiter; De Beni, \& Cornoldi, 2010; De Beni, Cornoldi, Carretti, \& Meneghetti, 2003; Kintsch \& Rawson, 2005; López-Escribano, Elosua de Juan, Gómez Veiga, \& García Madruga, 2013).

La escritura académica requiere, a su vez, del dominio progresivo de las formas y funciones avanzadas del lenguaje, implicadas en la composición de estructuras mayores a la palabra (Cubo de Severino, 2006). Ellas involucran procesos de planificación de las ideas que se quieren trasmitir, la redacción o traducción de tales ideas en oraciones ordenadas adecuadamente entre sí y la revisión del texto para comprobar si expresa adecuadamente las intenciones del escritor (Fayol, 2007; Hayes, 2006, Levy \& Ransdell, 2006).

Tanto la lectura como la escritura se despliegan en relación con un texto a comprender o producir, en función del objetivo perseguido (Fayol, 1996). De acuerdo con ello los factores que influyen en la elaboración pueden variar considerablemente. No obstante, en ambos casos, ha sido señalada reiteradamente la importancia de la metacognición de los comprensores/escritores en dichas tareas (Mokhtari \& Reichard, 1996). Se pueden distinguir al menos dos aspectos que componen la metacognición: por un lado el conocimiento de los procesos cognitivos o la conciencia de los procesos cognitivos, y por otro, la regulación y el control de dichos procesos (Marti, 1995; Costabile, Cornoldi, De Beni, Manfredi, \& Figluzzi, 2013). El primer aspecto refiere a un componente declarativo, al "saber qué", e incluye tareas tan variadas como darse cuenta de la dificultad de una tarea, saber que una persona explica mejor que otra, conocer las propias capacidades para determinada tarea, etc. El segundo, a un "saber cómo" o saber procedimental e incluye la posibilidad de tomar precauciones ante una tarea ardua, regular el esfuerzo y el tiempo que requiere según su dificultad, entre otros. La relación de ambos aspectos es de una interdependencia marcada (Urquijo, 2002).

Entre las múltiples investigaciones a propósito de los procesos involucrados en la comprensión lectora (Polselli Sweet \& Snow, 2003), y en la producción escrita (Fayol, 2007; Harp, 2007; MacArthur, Graham, \& Fitzgerald, 2006) algunas tendencias relativamente recientes acuerdan en el rol preponderante que juega la conciencia metacognitiva, tanto en los procesos motivacionales como cognitivos mientras se lee o escribe (Mokthari \& Reichard, 2002, Escoria, 2010; García Guzmán \& Salvador Mata, 2006; González \& Tourón; 1992). Efectivamente, uno de los aspectos metacognitivos de mayor interés corresponde al autoconocimiento que tiene un sujeto al momento de realizar una tarea, en este caso las tareas de lectura y escritura (Bong \& Skaalvik, 2003; Carlino, 2005; Costabile et al., 2013; Mateos, 2001, Schmidt, Messoulam, \& Molina, 2008). En la bibliografía especializada 
aparecen dos conceptos que se refieren a este dominio: el autoconcepto y la autoeficacia percibida.

Si bien el termino autoconcepto (Casullo, 1990) tiene una larga tradición en la historia de la Psicología, desde la Psicología Cognitiva del procesamiento de la información puede definirse como una estructura de conocimiento del sí mismo, y como un proceso que influye en todas las fases del procesamiento de la información (González \& Tourón, 1992). Por su lado, la autoeficacia se refiere a la percepción que un individuo tiene de su capacidad o su habilidad para realizar una determinada tarea, siendo esta percepción predictora de su conducta (Garcia Guzman \& Salvador Mata, 2006).

El autoconcepto es entonces una percepción más global que el sujeto construye en torno a su persona mientras que la autoeficacia percibida se refiere a una percepción más acotada que el sujeto construye sobre sí mismo, en áreas de actuación específicas (González \& Tourón, 1992). Si bien algunos autores señalan la importancia de diferenciar el autoconcepto de la autoeficacia percibida (Zimmerman, 2000), otros sostienen que las percepciones de autoeficacia en una tarea, en este caso de lectura y escritura, son la base cognitiva para el desarrollo del autoconcepto en ese dominio (Bong \& Skaalvic, 2003). El mismo será fundamental en la regulación posterior de las prácticas de lectura y escritura (Escoria, 2010, Abusamra et al., 2011; Ugartetxea, 2002).

Más allá de las precisiones en torno a ellos, resulta llamativo el menor número de trabajos en torno a la autopercepción de los alumnos en su desempeño en lectura y escritura.

En función de lo expuesto, resulta de interés conocer la perspectiva de los alumnos universitarios sobre este tema, como una de las fuentes de información destinada a diseñar propuestas de intervención que tiendan optimizar su desempeño a la vez que disminuir el abandono universitario. En función de ello, el objetivo del presente trabajo, como ya se señalara, es justamente describir el modo en que se autoperciben en tanto lectores y escritores alumnos de la Licenciatura en Psicología. Se trata de indagar si reconocen tener dificultades y en tal caso cuáles son las que detectan, así como las causas que atribuyen a sus desempeños en ambas actividades. Para ello se examinaron las características que sobre esos aspectos presentan los alumnos en dos tramos de su trayecto formativo: al comenzar la carrera y al cursar el tramo final de la misma. Resulta relevante identificar si existen características diferenciales o no entre los dos grupos en lo relativo a distintos indicadores seleccionados, considerando que en los años superiores las dificultades percibidas deberían ser menores y mayor la posibilidad de identificarlas, cuando persisten algunas, así como atribuir las causas plausibles a las que obedecen.

\section{Método}

\section{Diseño}

Se trata de un estudio transeccional descriptivo (Hernández Sampieri, Fernández Collado, \& Baptista Lucio, 2010) donde se analizan las características de las variables seleccionadas, en estudiantes universitarios en dos tramos del trayecto formativo.

\section{Participantes}

Se seleccionó una muestra intencional de 50 alumnos de ambos sexos de 18 a 21 años de edad que cursaban el primer año y 40 alumnos de ambos sexos de 23 a 27 años de edad, que cursaban el quinto año de la Carrera Licenciatura en Psicología.

\section{Instrumentos}

Se administró un cuestionario elaborado específicamente para este trabajo sobre autopercepción del desempeño en comprensión y producción textual, sobre: a) la presencia/ausencia de dificultades en relación con la fluidez lectora, la comprensión lectora y las actividades redaccionales; b) el tipo de dificultades que pueden presentarse y c) la atribución causal de las mismas (ver Anexo). El mismo fue sometido al criterio de 6 jueces expertos, sobre la pertinencia de los indicadores considerados, que arrojó una coincidencia positiva alta.

\section{Procedimientos}

\section{Obtención de datos}

Los alumnos fueron examinados colectivamente con el instrumento seleccionado, en dependencias de la Facultad de Psicología, de la Universidad Nacional de la Plata (Argentina). En el caso de los alumnos de primer año la administración del cuestionario se realizó en la cursada regular de la asignatura Psicología I y los de quinto año, en la cursada regular de la materia Psicología Educacional. Se explicaron los términos involucrados en el cuestionario, aclarándose que ante cualquier duda el examinador procedería a aclararla. Todos dieron su consentimiento informado para tal 
participación, así como para la publicación de los resultados, asegurándoles el anonimato de los mismos.

\section{Análisis y elaboración de los datos}

Los datos obtenidos fueron clasificados y posteriormente volcados a una base de datos, para su procesamiento estadístico (Decision Analyst STATS TM, 2.0). Se calcularon los porcentajes de respuestas en cada uno de los indicadores incluidos en las variables seleccionadas. Luego se procedió a calcular la significación de las diferencias entre porcentajes para realizar la comparación de los datos obtenidos en los dos grupos de alumnos examinados.

\section{Resultados}

\section{Presencia/ausencia de dificultades. Tipo lector/escritor}

Lectura. El $30 \%$ de los participantes de quinto año expresa no tener dificultades en la lectura, mientras que en primer año el porcentaje desciende al $10 \%$. Esa diferencia alcanza significación estadística $(p>0,016)$, situación que informa que los alumnos más avanzados tienen una percepción mayor de problemas en este dominio.

La indagación de la autopercepción del tipo lector de los estudiantes de $5^{\circ}$ año evidencia que la mayoría considera que lee con fluidez (precisión, velocidad y prosodia adecuada al leer en voz alta). No obstante, el 15\% informa tenerlas. Entre los estudiantes de primer año, ese porcentaje asciende a algo más de la mitad. Las diferencias entre ambos grupos alcanzan significación estadística, de modo tal que, como es esperable, los alumnos de años superiores se perciben como lectores más fluidos.

Más problemática resulta la comprensión de textos, ya que la mayoría de los alumnos de $5^{\circ}$ año manifiesta comprender el significado del texto sólo en algunas oportunidades, mientras sucede lo contrario en muchas otras. En el mismo sentido casi la totalidad de los de $1^{\circ}$ año responde tener algunas o muchas dificultades. Las diferencias entre ambos grupos alcanzan significación estadística, dando cuenta de problemas en los dos, pero de una autopercepción mayor de dificultades entre los alumnos que están iniciando la carrera.

Escritura. Cuando se trata de la escritura, los alumnos de ambos grupos informan no tener dificultades aproximadamente en la misma proporción (20\% en los de $1^{\circ}$ año y $25 \%$ en los de $5^{\circ}$ año). Cabe señalar que mientras el porcentaje de los de $1^{\circ}$ año es el doble respecto de la lectura, en los de $5^{\circ}$ es algo menor.

$\mathrm{Si}$ bien las diferencias en redacción no alcanzan significación estadística entre ambos grupos, los de $1^{\circ}$ año reportan tener menores dificultades que los de $5^{\circ}$ (aproximadamente la mitad de los que cursan $5^{\circ}$ año y algo menos de la mitad de los que cursan $1^{\circ}$ ) (Tabla 1 ).

Tabla 1. Autopercepción del tipo lector y escritor en estudiantes de primero y quinto año

\begin{tabular}{|c|c|c|c|c|}
\hline \multirow{2}{*}{ Indicadores } & \multicolumn{2}{|c|}{ Con dificultades } & \multirow[t]{2}{*}{$\mathrm{z}$} & \multirow[t]{2}{*}{$p$} \\
\hline & $1^{o}$ & $5^{\circ}$ & & \\
\hline Fluidez Lectora & $58 \%$ & $15 \%$ & 4,158 & $.000 *$ \\
\hline $\begin{array}{l}\text { Comprensión } \\
\text { lectora }\end{array}$ & $94 \%$ & $72,50 \%$ & 2,121 & $.005 *$ \\
\hline Redacción & $38 \%$ & $50,00 \%$ & 1,142 & .253 \\
\hline
\end{tabular}

Tipo de dificultades percibidas

Lectura. Las dificultades en lectura mayoritarias informadas por los alumnos de ambos grupos resultan estadísticamente significativas respecto del vocabulario y la detección de ideas principales y secundarias, en favor de porcentajes menores en los dos indicadores en los de $5^{\circ}$ año. En cuanto a la realización de inferencias y a la metacomprensión, no aparecen diferencias intergrupales. Llama la atención que aproximadamente un tercio del total de los alumnos no perciban tener dificultades en la realización de inferencias, y que resulte exiguo el porcentaje de aquellas atribuibles a la metacomprensión (Tabla 2).

Tabla 2. Autopercepción del tipo de dificultades en lectura en estudiantes de primero y quinto año

\begin{tabular}{|c|c|c|c|c|}
\hline \multirow[t]{2}{*}{ Indicadores } & \multicolumn{2}{|c|}{$\begin{array}{c}\text { Con } \\
\text { dificultades } \\
\end{array}$} & \multirow[t]{2}{*}{ Z } & \multirow[t]{2}{*}{$p$} \\
\hline & $1^{\circ}$ & $5^{\circ}$ & & \\
\hline Vocabulario & $58 \%$ & $27,5 \%$ & 2,893 & $.003 * *$ \\
\hline $\begin{array}{l}\text { Ideas } \\
\text { principales/secundarias }\end{array}$ & $30 \%$ & $12,5 \%$ & 1,984 & $.047 *$ \\
\hline Inferencias & $26 \%$ & $27,5 \%$ & 0,160 & .873 \\
\hline Metacomprensión & $18 \%$ & $10 \%$ & 1,073 & .288 \\
\hline
\end{tabular}

Escritura. En cuanto al tipo de dificultades que detectan en la escritura, ninguna excede al $30 \%$ y no aparecen diferencias significativas entre ambos grupos en ninguno de los indicadores considerados. Las percibidas, mayoritariamente se sitúan en el vocabulario y en la claridad y la precisión para redactar. Además un porcentaje de 
alumnos de $5^{\circ}$ año menciona el desconocimiento de la estructura textual y el estilo propio del lenguaje escrito como dificultad. Es muy baja la autopercepción de dificultades referidas a la adecuación al destinatario (Tabla 3).

\begin{tabular}{|c|c|c|c|c|}
\hline \multirow[t]{2}{*}{ Indicadores } & \multicolumn{2}{|c|}{$\begin{array}{c}\text { Con } \\
\text { dificultades }\end{array}$} & \multirow[t]{2}{*}{$\mathrm{Z}$} & \multirow[t]{2}{*}{$p$} \\
\hline & $1^{\circ}$ & $5^{\circ}$ & & \\
\hline Ortografía & $16 \%$ & $10 \%$ & 0,832 & .405 \\
\hline Vocabulario & $28 \%$ & $22,5 \%$ & 0,594 & .552 \\
\hline Gramática & $4 \%$ & $12,5 \%$ & 1,496 & .136 \\
\hline $\begin{array}{l}\text { Claridad y } \\
\text { precisión }\end{array}$ & $30 \%$ & $27,5 \%$ & 0,260 & .749 \\
\hline $\begin{array}{l}\text { Estructura } \\
\text { textual }\end{array}$ & $10 \%$ & $20 \%$ & 1,341 & .179 \\
\hline $\begin{array}{c}\text { Adecuación al } \\
\text { destinatario }\end{array}$ & $6 \%$ & $2,5 \%$ & 0,801 & .923 \\
\hline Estilo & $18 \%$ & $15 \%$ & 0,379 & .704 \\
\hline
\end{tabular}

\section{Atribución causal del desempeño en lectura y escritura}

Las opciones a señalar respecto de la atribución causal de las dificultades percibidas arrojan un abanico de respuestas en ambos grupos, con altos porcentajes en algunos de los indicadores considerados.

Lectura. Con respecto a la lectura, resulta de interés señalar que en general solo aparecen diferencias significativas entre los alumnos $\mathrm{d} 1^{\circ} \mathrm{y}$ $5^{\circ}$ año cuando se trata de conocimientos previos sobre el tema, posibilidad de hacer inferencias, conocimiento de las estructuras textuales y estado de ánimo, en favor de porcentajes más altos en los alumnos más avanzados (Tabla 4).

Escritura. En la escritura los porcentajes de respuesta son semejantes en ambos grupos de alumnos. En general atribuyen mucha importancia a cada uno de las alternativas proporcionadas. Solo se observan diferencias significativas cuando se trata de indicadores referidos al estado de ánimo, a la disponibilidad de bibliografía de consulta y a la enseñanza de los profesores, a los que los alumnos de $5^{\circ}$ año les atribuyen mayor importancia (Tabla 5).

Tanto en lectura como en escritura es llamativo el porcentaje de respuestas referido a dificultades atribuibles a la posibilidad de estudiar o escribir con compañeros y a la inteligencia, cuyos guarismos se encuentran por debajo de los demás.

\section{Conclusiones}

Este trabajo estuvo destinado a examinar la autopercepción como lectores y escritores de los estudiantes de $1^{\circ}$ y $5^{\circ}$ año de la carrera de Psicología. Como se recordará se indagaron para ambas variables el tipo lector, referido a la presencia/ausencia de dificultades, a la fluidez, comprensión lectora y redacción, el tipo de dificultades en distintos indicadores en ambas variables y la atribución causal de las mismas.

Del análisis de los resultados obtenidos se concluye que tales autopercepciones cambian según los tramos formativos considerados sólo en algunos de los indicadores considerados. En líneas generales, es esperable el mayor porcentaje de respuestas de alumnos de $5^{\circ}$ año que informan la autopercepción de ausencia de dificultades, habida cuenta de su avance en la carrera, no obstante lo cual aproximadamente un tercio de ellos afirma percibirlas. El porcentaje de respuestas de los dos grupos considerados, que indican ausencia de dificultades es bajo: en lectura el $10 \%$ de los de $1^{\circ}$ año y el $30 \%$ de los de $5^{\circ}$ en tanto en escritura los porcentajes corresponden al $20 \%$ y al $25 \%$ respectivamente. Estos datos indican de modo sucinto que las dificultades de escritura percibidas por los alumnos son mayores que los de la lectura entre los de $1^{\circ}$ año y menores entre los de $5^{\circ}$ año.

Se recordará que fundamentalmente en el campo de la lectura, se tomaron como indicadores globales la fluidez lectora y la comprensión lectora. Mientras el $58 \%$ de los alumnos de $1^{\circ}$ año declara tener dificultades en fluidez, situación a ser considerada en relación con posibles causas de deserción en los estudios, ese porcentaje desciende al $15 \%$ en los alumnos de $5^{\circ}$ año. Las diferencias, que alcanzan significación estadística, ilustran sobre una situación crítica entre los ingresantes, pero al mismo tiempo no deja de llamar la atención el porcentaje de alumnos avanzados que aun autoperciben dificultades. Cabe señalar al respecto que en este tramo del trayecto formativo no es dable esperar dificultades de ese tipo en ninguno de los alumnos, ya que, tal como ha sido indagado en este trabajo, la fluidez concierne a la velocidad, precisión y prosodia adecuada en la lectura en voz alta. Cabría esperar en tal sentido menos dificultades entre los principiantes y ninguna entre los próximos a egresar, como se señala en la literatura especializada, cuando se caracteriza la progresión del desempeño en ambas variables, una vez 
Tabla 4. Importancia atribuida a las posibles causas del propio desempeño en lectura en estudiantes de primer y quinto año

\begin{tabular}{lcccc}
\hline \multirow{2}{*}{ Indicadores } & \multicolumn{4}{c}{ Muy importante/importante } \\
\cline { 2 - 5 } & $1^{\mathbf{0}}$ & $5^{\mathbf{0}}$ & $\mathrm{z}$ & $p$ \\
\hline Esfuerzo & $98 \%$ & $90 \%$ & 1.646 & .099 \\
Inteligencia & $66 \%$ & $70 \%$ & 0,403 & .686 \\
Conocimientos previos sobre el tema & $42 \%$ & $82,5 \%$ & 3,897 & $.000^{*}$ \\
Posibilidad de hacer inferencias & $42 \%$ & $95 \%$ & 5,258 & $.000^{*}$ \\
Conocimiento de las estructuras textuales & $38 \%$ & $92,5 \%$ & 5,299 & $.000^{*}$ \\
Motivación para estudiar & $98 \%$ & $100 \%$ & 0,899 & .386 \\
Propósitos que se persiguen al leer & $90 \%$ & $95 \%$ & 0,880 & .378 \\
Estado de ánimo & $84 \%$ & $97,5 \%$ & 2,121 & $.033 * *$ \\
Dificultad del texto & $92 \%$ & $95 \%$ & 0,567 & .570 \\
Disponibilidad bibliografía y lugar & $92 \%$ & $90 \%$ & 0,331 & .740 \\
Explicación de los profesores & $98 \%$ & $95 \%$ & 0,788 & .430 \\
Posibilidad de estudio con compañeros & $62 \%$ & $75 \%$ & 0,311 & .189 \\
\hline
\end{tabular}

finalizados los estudios secundarios y cuando se cursan los años superiores de los universitarios (Harp, 2007). Respecto de la comprensión, se observa el mismo perfil. Obviamente, aunque el porcentaje de dificultades percibidas entre los alumnos de $5^{\circ}$ año es menor respecto de los de $1^{\circ}$ $(72,50 \%$ vs. $94 \%)$, no deja de ser llamativo, dado que informa que los problemas de comprensión aún están presentes para muchos de ellos. Sin embargo esta información debe matizarse ya que el grupo de estudiantes más avanzado mayoritariamente informó que algunas veces comprende y otras no.

En relación con las habilidades redaccionales los alumnos de quinto año manifiestan tener más dificultades que los de $1^{\circ} \quad(50 \%$ y $38 \%$ respectivamente), no obstante lo cual es relativamente alto el porcentaje en ambos casos. Esto puede deberse a la escasa práctica de escritura que tienen los primeros al iniciar la carrera, práctica necesaria para dimensionar y conceptualizar las dificultades en ese dominio. Pero igualmente llaman la atención las que persisten entre los alumnos de $5^{\circ}$ año, situación que tangencialmente informa sobre la insuficiencia de los aprendizajes a pesar de lo avanzado de la carrera.

En las respuestas obtenidas en relación con el tipo de dificultad percibida, tanto en lectura como en escritura, aparecen diferencias significativas entre los porcentajes de respuestas proporcionados por ambos grupos solo en algunos de los indicadores considerados. Los resultados encontrados ponen de manifiesto conceptualizaciones que pueden sumarizarse de la siguiente manera.

Las dificultades de lectura, que resultan significativamente diferentes, refieren al conocimiento del vocabulario (caudal léxico) y a la detección de ideas principales y secundarias. Es mayor entre los alumnos de $1^{\circ}$ año respecto de $5^{\circ}(58 \%$ y $27,5 \%$ y $58 \%$, y $30 \%$ y $12,5 \%$ respectivamente). No obstante, es llamativa la persistencia del reporte de la percepción de tales dificultades de parte de los alumnos más avanzados, aún en habilidades relativamente básicas como las que se ponen de manifiesto a través de esos indicadores. En la producción de inferencias, es remarcable el porcentaje semejante y relativamente bajo ( $26 \%$ en $1^{\circ}$ año y $27,5 \%$ en $5^{\circ}$ año) de aquellos que expresan tenerlas, siendo éste un componente crítico en la comprensión textual. Asimismo llama la atención que los alumnos de ambos grupos perciban en un porcentaje relativamente exiguo dificultades en la detección de su propia comprensión (metacomprensión) (18\% en $1^{\circ}$ año y $10 \%$ en $5^{\circ}$ año), en relación con las dificultades reales que se observan que aparecen cuando deben interpretar el significado de un texto.

En la escritura no aparecen diferencias significativas entre los porcentajes de respuestas de los dos grupos, y en ningún caso exceden al $30 \%$. Es de señalar que cuando se trata del vocabulario los porcentajes de dificultades percibidas son menores que los reportados en la lectura. Son más acentuadas entre los de $1^{\circ}$ año ( $28 \%$ vs. $58 \%$ ), y menor entre los de $5^{\circ}$ año $(22,5 \%$ vs. $27,5 \%)$. Esto posiblemente pueda atribuirse a que existen diferencias entre el conocimiento del vocabulario pasivo y activo. Es decir, entre aquel que se conoce cuando se lee y aquel que debe evocarse cuando se escribe (Segui \& Ferrand, 2000). Otra de las dificultades percibidas en mayor medida en la escritura corresponde a la claridad y precisión en la 
Tabla 5. Importancia atribuida a las posibles causas del propio desempeño en escritura en estudiantes de primer y quinto año

\begin{tabular}{lcccc}
\hline \multirow{2}{*}{ Indicadores } & \multicolumn{4}{c}{ Muy Importante/importante } \\
\cline { 2 - 5 } & $1^{\circ}$ & $5^{\circ}$ & $\mathrm{z}$ & $p$ \\
\hline Esfuerzo & $88 \%$ & $87.5 \%$ & 0,072 & .942 \\
Inteligencia & $78 \%$ & $75 \%$ & 0,334 & .738 \\
Conocimientos previos sobre el tema & $92 \%$ & $90 \%$ & 0,331 & .740 \\
Conocimiento de las estructuras textuales & $90 \%$ & $92,5 \%$ & 0,414 & .678 \\
Motivación para escribir & $98 \%$ & $100 \%$ & 0,899 & .368 \\
Propósitos que se persiguen al escribir & $94 \%$ & $95 \%$ & 0,206 & .836 \\
Estado de ánimo & $80 \%$ & $95 \%$ & 2,080 & $.037 * *$ \\
Dificultad del texto & $90 \%$ & $92,5 \%$ & 0,414 & .678 \\
Disponibilidad bibliografía de consulta & $84 \%$ & $97,5 \%$ & 2,121 & $.033^{* *}$ \\
Explicación de los profesores & $98 \%$ & $87,5 \%$ & 1,984 & $.047^{* *}$ \\
Posibilidad de escribir con compañeros & $56 \%$ & $72,5 \%$ & 1,614 & .104 \\
\hline$* * p>.05$ & & & &
\end{tabular}

exposición de las ideas. Aparecen porcentajes relativamente importantes si se considera que en la escritura resultan relevantes en la composición del texto extenso. Se destaca que no se observa una disminución en la percepción de dificultades de este tipo en los alumnos más avanzados. Respecto de la estructura textual, es bajo el porcentaje de dificultades reportadas por ambos grupos, aunque se duplica el de los alumnos de $5^{\circ}$ año (20\%) respecto de los de $1^{\circ}$ (10\%). Posiblemente esto pueda relacionarse con el mayor conocimiento de los primeros respecto de la estructura textual, en razón de la experiencia que tienen con esas estructuras por las distintas prácticas de lectura y escritura a medida que se avanza a lo largo de la carrera, que no obstante no aparecen suficientemente dominadas. Respecto del estilo y la ortografía, los porcentajes de repuesta son semejantes, aunque en una proporción algo menor que las proporcionadas a otros indicadores de la variable. Los porcentajes de respuestas sobre las dificultades percibidas en relación con la gramática, son muy bajos en ambos casos, pero significativamente mayores en los alumnos de $5^{\circ}$ año $(12,5 \%)$ respecto de los de $1^{\circ}(4 \%)$, situación también adjudicable al mayor conocimiento que poseen sobre las características y restricciones que operan en el texto extenso.

En su conjunto, la autopercepción de tales dificultades coincide con los resultados de otras investigaciones que ponen en evidencia las dificultades que presentan los alumnos aun en este nivel. En ellas se informan que suelen persistir aquella inherentes a una pluralidad de dominios: información general inadecuada, concepciones erróneas (misconceptions), tratamiento insuficiente de textos complejos $y$ desconocimiento de términos generales y específicos (McMahon \& McCormack, 1998;
Piacente, Fernícola, \& Piatti, 2010). Cabe señalar al respecto que en este tramo del trayecto formativo no es dable esperar dificultades de ese tipo en ninguno de los alumnos. Persisten al respecto algunos interrogantes sobre los factores responsables de esta situación, factores considerados por los mismos alumnos, además de otros, que han sido tratados en diferentes investigaciones (Kintsch, \& Rawson 2005, Zabaleta, 2014).

Respecto de la atribución causal de las dificultades autopercibidas, aparecen diferencias significativas entre los alumnos de $1^{\circ}$ y $5^{\circ}$ año en algunos de los indicadores de la lectura o la escritura.

Cuando se trata de la lectura, esas diferencias corresponden a los conocimientos previos sobre el tema, a la posibilidad de hacer inferencias, al conocimiento de las estructuras textuales y al estado de ánimo. Se destaca que, excepto en este último indicador, los porcentajes de respuestas proporcionados por los alumnos más avanzados duplican a los de los ingresantes. Sobre los mismos existe consenso entre los especialistas en el tema acerca del papel relevante que cumplen en la comprensión textual (Kintsch, \& Rawson; 2005). Esta situación conduce a plantear que a medida que los alumnos avanzan en la carrera tienen mayores conocimientos del papel que juegan tales indicadores. En cuanto al estado de ánimo es razonable pensar que está ligado a consideraciones de tipo emocional, vinculadas con las exigencias propias de la actividad académica. Es decir que, a medida que se progresa en los estudios se informan en mayor proporción las causas relacionadas con la posibilidad de vincular las informaciones proporcionadas por el texto con aquellas que dependen del conjunto de conocimientos previos (generales del mundo, 
específicos de dominio y conocimientos lingüísticos), de reponer información o vincular aquella que aparecen distante en el mismo texto, de la dependencia de la comprensión lectora del conocimiento de la estructura textual y de la situación emocional que puede acompañar a la tarea de leer.

Cabe señalar, que tanto un grupo como otro han seleccionado al papel que cumple la inteligencia y la posibilidad de estudio con otros compañeros en un porcentaje menor, aunque relativamente alto (entre el $60 \%$ y el $70 \%$ ) respecto de los otros indicadores.

En las posibles causas de dificultades en escritura, prácticamente se homologa el alto porcentaje de respuestas proporcionadas por los alumnos de ambos grupos: casi todos los indicadores son señalados como de importancia. Pero también es menor el porcentaje de repuestas sobre la inteligencia y la posibilidad de escribir con otros compañeros.

Según estos resultados los alumnos perciben con diferente grado de intensidad las dificultades que enfrentan cuando deben comprender o producir un texto. Razonablemente puede sostenerse, además, que la autopercepión de los alumnos como lectores y escritores presenta algunas características diferenciales a medida que avanzan en la carrera. Efectivamente las respuestas varían según se trate del desempeño lector/escritor, del tipo de dificultades y de la atribución causal de las mismas. La totalidad de ellos responde tener algún grado de dificultad en cada uno de los indicadores de las variables de lectura y escritura seleccionados. Recuérdese al respecto que las opciones disponibles respecto de la atribución causal no son mutuamente excluyentes, de modo tal que un mismo alumno puede señalar dificultades en uno o más de esos indicadores. Sin embargo sería necesaria una investigación más exhaustiva sobre aquello que manifiestan acerca del alcance de cada una de las opciones ofrecidas. De todos modos resulta de utilidad contar con estas informaciones a los efectos de tomarlas en consideración en las estrategias de enseñanza, tanto en aquellas que apoyen a los aspectos en los que los alumnos perciben tener mayores dificultades, como en el esclarecimiento de los alcances de los indicadores, en los que los porcentajes de respuesta son exiguos y sin embargo juegan un papel crucial en la interacción textual.

De particular importancia aparece la estimación del tipo de dificultades en escritura en relación con las de lectura en ambos grupos, situación que contrasta con el desempeño efectivo observado en otras investigaciones.

Otra cuestión de interés, cuando se trata del papel que juega la producción de inferencias en la lectura, ligada, obviamente, a los conocimientos previos, y a la metacomprensión, que permite monitorear el proceso lector, es la aparición de un porcentaje relativamente bajo de respuestas de ambos grupos que indican dificultades de ese tipo $\left(26 \%\right.$ en $1^{\circ}$ año y $27,5 \%$ en $5^{\circ}$ año). Contrariamente es alto el papel que le atribuyen como causa, mayor en el caso de los alumnos avanzados ( $42 \%$ en $1^{\circ}$ año y $95 \%$ en $5^{\circ}$ año). Esto puede significar que si bien los alumnos tienen conciencia de los factores que afectan el desempeño en comprensión lectora, no perciben dichas dificultades como propias.

Finalmente si bien la autopercepción de los alumnos de $5^{\circ}$ año refleja la posibilidad de reflexión mayor frente a su desempeño lector/escritor, no deja de ser significativo que aún aparezcan respuestas que problematizan su situación.

El conjunto de los resultados obtenidos conduce, por un lado, a la necesidad de contrastar las informaciones obtenidas sobre la autopercepción de los alumnos como lectores/escritores con su desempeño efectivo, situación que ha sido examinada en otras investigaciones (Olave Arias, Rojas García \& Cisneros Estupiñán, 2013; Piacente \& Tittarelli, 2008), y que se proseguirá con los mismos alumnos. Por otro, pone de relieve la necesidad de tomar en consideración los aspectos examinados de modo tal de incluir la perspectiva de los propios alumnos frente a la posibilidad de un interacción textual exitosa, a la hora de diseñar estrategias de orientación e intervención. Efectivamente tanto la comprensión lectora, que facilita el aprendizaje a partir de los textos, cuanto la producción escrita, por su papel epistémico, resultan instrumentos indispensables en cualquier disciplina universitaria y los esfuerzos en pos de la alfabetización académica no pueden dejar de lado la necesidad de mejorar todos los aspectos metacognitivos concernidos en ambos dominios, cuyo papel ha sido reconocido en variadas investigaciones.

\section{Referencias}

Abusamra, V., Ferreres, A., Raiter, A., De Beni. R., \& Cornoldi, C. (2010). Test Leer para comprender: Evaluación de la comprensión de textos. Buenos Aires: Editorial Paidos. 
Abusamra, V. Casajús, A. Ferreres, A. Ratier, A. De Beni, R., \& Cornoldi, C. (2011). Programa Leer para Comprender. Libro Teórico. Buenos Aires: Paidos.

August, D., \& Shanahan, T. (Eds.) (2008). Developing Reading and Writing in SecondLanguage Learners: Lessons From the Report of the National Literacy Panel on LanguageMinority Children and Youth. New York: Routledge.

Bong, M., \& Skaalvik, E. (2003). Academic selfconcept and self-efficacy. How different are they really? Educational Psychology Review 15(1), 1-40.

Casullo, M. M. (1990): El autoconcepto. Técnicas de evaluación. Buenos Aires: Psicoteca Editorial.

Carlino, P. (2005). Escribir, leer, y aprender en la universidad. Una introducción a la alfabetización académica. Buenos Aires: Fondo de Cultura Económica.

Costabile, A., Cornoldi, C., De Beni, R., Manfredi, P., \& Figliuzzi, S. (2013). Metacognitive components of student's difficulties in the first year of university. International Journal of Higher Education, 2(4), 165-171.

Cubo de Severino, L. (Coord.) (2007). Los textos de la Ciencia. Principales clases del discurso académico-científico. Córdoba (Argentina): Comunicarte.

De Beni, R., Cornoldi, C., Carretti, B., \& Meneghetti, B. (2003). Unova guida alla comprensione del testo. Trento: Erickson

Escoria, D. (2010) Conocimientos metacognitivos y autorregulación: Una lectura cualitativa del funcionamiento de los estudiantes universitarios en la producción de textos. Avances en Psicología Latinoamericana, 28(2), 265-277.

Fayol, M. (1996). A propósito de la comprensión... Les journées de l'ONL, Regarde sur la lecture et ses apprentissages. Disponible on line http://onl.inrp.fr.

Fayol, M. (2007). La production de textes et son apprentissage. Les journées de l'ONL, Écrire des textes, l'apprentissage et le plaisir. Disponible on line http://onl.inrp.fr.
García Guzmán, A., \& Salvador Mata, F. (2006). La autopercepción de eficacia en la lectura: Una revisión de investigaciones. Enseñanza, 24, 79-99.

González, M. C., \& Tourón, J. (1992). Autoconcepto y rendimiento escolar. Sus implicaciones en la motivación y en la autorregulación del aprendizaje. Pamplona: EUNSA

Halliday, M. A. K., \& Martin, J. R. (1993). Writing science: Literacy and discursive power. Pittsburgh: University of Pittsburgh Press.

Harp, B. (2007). The Handbook of Literacy Assessment and Evaluation. Norwood, Massachusetts: Christopher Gordon

Hayes, J.R. (2006). New directions in writing theory. In Ch. A. MacArthur, S. Graham, \& J. Fitzgerald (Eds.), Handbook of writing Research (pp. 28-40). New York: The Guilford Press.

Hernández Sampieri, M. C., Fernández Collado, C. \& Baptista Lucio, P. (2010). Metodología de la investigación. México: McGRAWHILL.

Kintsch, W., \& Rawson, K. A. (2005). In M. J. Snowling \& Ch. Hulme (Eds.), The Science of Reading: A Handbook, (pp.209-226). Malden, MA: Blackwell Publishing.

Levy, C. M., \& Ransdell, S. (Eds.) (1996). The science of writing: Theories, methods, individual differences and application. Mahwah, NJ: Lawrence Erlbaum.

López Escribano, C., Elosua de Juan, M. R., Gómez Veiga, I., \& García Madruga, J. A. (2013). A predictive study of Reading comprehension in thir grade Spanish students. Psicothema 25(2), 199-205. DOI: 10.7334/psicothema2012.175 •

Martí, E. (1995). Metacognición: Entre la fascinación y el desencanto. Infancia $y$ Aprendizaje, 72, 9-32

Mateos, M. (2001). Metacognicion y educación. Buenos Aires: Aique

MacArthur, C A., Graham, S., \& Fitzgerald, J. (Eds.) (2006). Handbook of writing research. New York: The Guilford Press.

McMahon, M. M., \& McCormack, B. B. (1998). To think and act like a scientist: Learning 
disciplinary knowledge. In C. R. Hynd (Ed.), Learning from Text Across Conceptual Domains (pp. 227-262). Mahwah, NJ: Lawrence Erlbaum Assocites Publishers.

Mokhtari, K., \& Reichard, C. A. (2002). Assessing students' metacognitive awareness of reading strategies. Journal of Educational Psychology, Vol 94(2), 249-259.

Olave-Arias, G., Rojas-García, I., \& CisnerosEstupiñán, M. (2013). Deserción universitaria y alfabetización académica. Educación y Educadores, 16(3), 455-471.

Piacente, T. (2012a). Alfabetización inicial y alfabetización académica. Investigación y Evaluación de los procesos implicados en el aprendizaje del lenguaje escrito. Revista Iberoamericana de Evaluación - e Avaliação Psicológica, 33(1), 9-30.

Piacente, T. (2012b). La comprensión verbal en alumnos universitarios. Orientación y Sociedad, 12, 57-70.

Piacente, Fernícola, \& Piatti (2010). Nivel de vocabulario en alumnos universitarios de psicología. Análisis preliminar. Trabajo presentado en el II Congreso Internacional de Investigación y Práctica Profesional en Psicología. XVII Jornadas de Investigación. Sexto Encuentro de Investigadores en Psicología del Mercosur. Facultad de Psicología de la UBA. Buenos Aires.

Piacente, T., \& Tittarelli, A. M. (2008). Comprensión y producción de textos en alumnos universitarios. La reformulación textual. Revista Internacional Orientación y Sociedad. V. 7, 125-134.

Polselli Sweet, A., \& Snow, C. E. (Eds.) (2003). Rethinking Reading Comprehension. New York: The Guilford Press.
Schleppegrell, M. J., \& Colombi, M. C. (Eds.) (2002). Developing advanced literacy in first and second languages: Meaning with power. Mahwah, NJ, Lawrence Erlbaum.

Segui, J., \& Ferrand, L. (2000). Leçons de parole. París: Editions Odile Jacob.

Schmidt, V., Messoulam, N., \& Molina, M. F. (2008). Autoconcepto académico en adolescentes de escuelas medias: Presentación de un instrumento para su evaluación. Revista Iberoamericana de Diagnóstico $y-e$ Avaliação Evaluación Psicológica, 25(1), 81106

Snow, C. E., \& Uccelli, P. (2009). The challenge of academic language. In Olson, D. R., \& N. Torrance (Eds.), The Cambridge Handbook of Literacy (pp. 112-133). Cambridge: Cambridge University Press.

Ugartetxea, J. (2002). La metacognición, el desarrollo de la autoeficacia y la motivación escolar. Revista de Psicodidactica, 13, 49-74.

Urquijo, S. (2002). Autoconcepto y desempeño académico en adolescentes. Relaciones con sexo, edad e institución. Psico-USF, 7, 205212.

Zableta, V. (2014). Los cambios en el desempeño en lectura y escritura en dos tramos del trayecto formativo. (Tesis Doctoral. Facultad de Psicología de la U.N.L.P.) Disponible on line: Repositorio Sedici http://sedici.unlp.edu.ar/handle/10915/39678. Universidad Nacional de La Plata.

Zimmerman, B. J. (2000). Self-Efficacy: An essential motive to learn. Contemporary Educational Psychology, 25(1), 82-91. 


\section{Anexo}

\section{CUESTIONARIO DE AUTOPERCEPCION DE LOS ALUMNOS UNIVERSITARIOS (LICENCIATURA EN PSICOLOGÍA-UNLP) COMO LECTORES/ESCRITORES}

\section{APELLIDO Y NOMBRE}

FECHA

EDAD

Hora de inicio:

\section{Instrucciones}

Por favor, lea atentamente cada una de las preguntas o enunciados que aparecen más abajo. Marque la respuesta u opción que más se ajusta a su opinión. En algunos casos puede marcar más de una opción.

¿Qué tipo de lector se considera?

1. No tengo dificultades, leo con fluidez, es decir con precisión, velocidad y recuperación de los elementos de entonación no presentes totalmente en el lenguaje escrito.

2. Leo con algunas dificultades de fluidez.

3. Leo con bastantes dificultades de fluidez.

¿Qué tipo de escritor se considera?

4. Redacto sin dificultades o con muy pocas dificultades.

5. Tengo algunos problemas para redactar.

6. Tengo muchos problemas para redactar.

Cuando escribe ¿Tiene algunas de las siguientes dificultades?

7. Tengo dificultades ortográficas (faltas de ortografía).

8. Tengo dificultades para encontrar el vocabulario adecuado.

9. Tengo dificultades para redactar de manera gramaticalmente correcta.

10. Tengo dificultades para exponer de manera clara y precisa lo que pretendo poner por escrito.

11. Tengo dificultades porque desconozco las estructuras de algunos textos (partes que lo componen) tales como monografías, informes, artículos científicos u otros).

12. Tengo dificultades para adecuar la escritura de acuerdo al destinatario.

13. Tengo dificultades para adecuar mi redacción de acuerdo al estilo con el que debe redactarse un informe, monografía, resumen, u otro texto académico.

14. No tengo dificultades.

Cuando leo textos académicos (textos para estudiar en la Facultad)

15. Comprendo todo adecuadamente.

16. Comprendo en algunas circunstancias, en otras no.

17. Tengo muchas dificultades de comprensión.

¿Tiene alguna de las siguientes dificultades para comprender lo que lee? Señale todas las opciones que considere necesarias.

18. Dificultades para comprender el significado de algunas palabras.

19. Dificultades para diferenciar apropiadamente las ideas principales y las secundarias.

20. Dificultades para hacer inferencias (completar la información que no aparece explícitamente en el texto).

21. Dificultades para evaluar su propia comprensión (advertir cuando comprende y cuando tiene dificultades).

22. Ninguna de ellas

En las diferentes asignaturas ¿Le han solicitado informes escritos?

23. Frecuentemente.

24. Con muy poca frecuencia

25. Nunca

En las diferentes asignaturas ¿Le han solicitado que escriba una monografía?

26. Frecuentemente.

27. Con muy poca frecuencia

28. Nunca 
En las diferentes asignaturas ¿Han utilizado evaluaciones escritas a través de preguntas abiertas (debe redactar las respuestas).

29. Frecuentemente.

30. Con muy poca frecuencia

31. Nunca

En las diferentes asignaturas ¿Han utilizado evaluaciones escritas a través de preguntas con respuestas de elección múltiple (múltiple choice) (debe buscar la respuesta correcta entre varias opciones)

32. Frecuentemente

33. Con muy poca frecuencia

34. Nunca

Los textos universitarios que he leído hasta la fecha

35. Mayoritariamente me han resultado motivadores/interesantes para estudiar

36. Sólo algunos pocos me han resultado motivadores/interesantes para estudiar

37. Mayoritariamente no me han resultado motivadores/interesantes para estudiar

38. Sólo algunos pocos no me han resultado motivadores/interesantes para estudiar

Marque con una $X$ la importancia que para Ud. tienen las siguientes posibles causas del desempeño en lectura. Debe seleccionar una de las tres opciones (muy importante, importante, poco importante) en todos los casos.

\begin{tabular}{|l|l|l|l|}
\hline \multicolumn{1}{|c|}{ Causas del desempeño en lectura } & $\begin{array}{c}\text { Muy } \\
\text { importante }\end{array}$ & Importante & $\begin{array}{c}\text { Poco } \\
\text { importante }\end{array}$ \\
\hline 39. Esfuerzo & & & \\
\hline 40. Inteligencia & & & \\
\hline 41. Conocimientos previos sobre el tema & & & \\
\hline 42. Posibilidad de hacer inferencias & & & \\
\hline 43. Conocimiento de las estructuras textuales & & & \\
\hline 44. Motivación para estudiar & & & \\
\hline 45. Propósitos que se persiguen al leer & & & \\
\hline 46. Estado de ánimo & & & \\
\hline 47. Dificultad del texto & & & \\
\hline 48. Disponibilidad bibliografía y lugar & & & \\
\hline 49. Explicación de los profesores & & & \\
\hline 50. Posibilidad de estudio con compañeros & & & \\
\hline
\end{tabular}

Marque con una $X$ la importancia que para Ud. tienen las siguientes posibles causas de su desempeño en escritura. Debe seleccionar una de las tres opciones (muy importante, importante, poco importante) en todos los casos.

\begin{tabular}{|l|l|l|l|}
\hline \multicolumn{1}{|c|}{ Causas del desempeño en escritura } & \multicolumn{1}{c|}{$\begin{array}{c}\text { Muy } \\
\text { importante }\end{array}$} & Importante & $\begin{array}{c}\text { Poco } \\
\text { importante }\end{array}$ \\
\hline 51. Esfuerzo & & & \\
\hline 52. Inteligencia & & & \\
\hline 53. Conocimientos previos sobre el tema & & & \\
\hline 54. Conocimiento de las estructuras textuales & & & \\
\hline 55. Motivación para escribir & & & \\
\hline 56. Propósitos que se persiguen al escribir & & & \\
\hline 57. Estado de ánimo & & & \\
\hline 57. Dificultad del texto & & & \\
\hline 59. Disponibilidad bibliografía de consulta & & & \\
\hline 63. Explicación de los profesores & & & \\
\hline 60. Posibilidad de escribir con compañeros & & & \\
\hline
\end{tabular}

\footnotetext{
${ }^{1}$ Completar la información proveniente del texto escrito con información de la que dispone el lector, no presente en el texto

${ }^{2}$ Conocimiento sobre la forma en que se organizan los contenidos de un texto.
} 